\title{
Formation of Particle Real Energy in the Bicubic Equation Limiting Particle Velocity Formalism with Possible Applications to Light Dark Matter
}

\author{
Josip Śoln ${ }^{1}$ \\ ${ }^{1}$ JZS Phys-Tech, Vienna, Virginia 22182, USA \\ Correspondence: Josip Śoln, JZS Phys-Tech, Vienna, Virginia 22182, USA. E-mail: jurasoln@ yahoo.com
}

Received: March 16, 2019

Accepted: March 27, 2019

Online Published: March 30, 2019

doi:10.5539/apr.v11n2p92

URL: https://doi.org/10.5539/apr.v11n2p92

\begin{abstract}
:
The complex particle energy, appearing in this article, with the suggestive choices of physical parameters,is transformed simply into the real particle energy. Then with the bicubic equation limiting particle velocity formalism, one evaluates the three particle limiting velocities, $c_{1}, c_{2}$ and $c_{3}$, (primary, obscure and normal) in terms of the ordinary particle velocity, $v$, and derived positive $m_{+}=m>0$ and negative $m_{-}=-m<0$ particle masses with $m_{+}^{2}=m_{-}^{2}=m^{2}$.

In general, the important quantity in solving this bicubic equation is the real square value $z^{2}(m)$ of the congruent parameter, $z(m)$, that connects real or complex value of particle energy, $E$, and the real or complex value of particle velocity squared, $v^{2}, 2 E z(m)=3 \sqrt{3} m v^{2}$. With real $z^{2}(m)$ one determines the real value of discriminant, $D$, of the bicubic equation, and they together influence the connection between $E$ and $v^{2}$. Hence, when $z^{2}<1$ and $D<0$ one has simply that $E \gg m v^{2}$. However,with $D \geq 0$ and $z^{2} \geq 1$, both $E$ and $v^{2}$ may become complex simultaneously through connecting relation $E=3 \sqrt{3} m v^{2} / 2 z(m)$, with their real values satisfying
\end{abstract}

$\operatorname{Re} E \succcurlyeq m\left(\operatorname{Rev}^{2}\right)$, keeping, however $z^{2}$ the same and real.

In this article, this new situation with $D \geq 0$ is discussed in detail.by looking as how to adjust the particle parameters to have $\operatorname{Im} E=0$ with implication that automatically also $\operatorname{Im} v^{2}=0$..In fact, after having adjusted the particle parameters successfully this way, one simply writes $\operatorname{Re} E=E$ and $\operatorname{Re}^{2}=v^{2}$. This way one arrives at that the limiting velocities satisfy $c_{1}=c_{2} \# c_{3}$, which shows the degeneracy of $c_{1}$ and $c_{2}$ as the same numerical limiting velocity for two particles. This degeneracy $c_{1}=c_{2}$ is simply due to the absence of $\operatorname{ImE}$. It would start disappearing with just an infinitesimal $\operatorname{ImE}$. Now,while $c_{1}=c_{2}$ is real, $c_{3}$ is imaginary and all of them associated with the same particle energy, $E$. With these velocity values the congruent parameter becomes quantized as $z\left(m_{ \pm}\right)=3 \sqrt{3} m_{ \pm} v^{2} / 2 E= \pm 1$ which, with the bicubic discriminant $D=0$ value, implies the quantization also of the particle mass, $m$, into $m_{ \pm}= \pm m$ values. The numerically equal energies,from $E=R e E$ can be expressed as

$E\left(c_{1,2}\left(m_{ \pm}\right)\right)=E\left(c_{3}\left(m_{ \pm}\right)\right)$either directly in terms of $c_{1}\left(m_{ \pm}\right)=c_{2}\left(m_{ \pm}\right)$and $c_{3}\left(m_{ \pm}\right)$or also indirectly in terms of particle velocity, $v$, as well as in the Lorentzian fixed forms with $v^{2} \# c_{1}^{2}, c_{2}^{2}$ or $c_{3}^{2}$ assuring different from zero mass, $m$ \# 0 .

At the end, with here developed formalism, one calculates for a light sterile neutrino dark matter particle, the energies associated with $m_{ \pm}$masses and $c_{1,2}$ and $c_{3}$ limiting velocities.

Keywords: positive and negative particle masses, dark matter particles, bicubic equations, limiting velocities

\section{Introduction}

The discussion of particle limiting velocities is done rather efficiently with the bicubic equation limiting particle velocity formalism as developed in Śoln (2014, 2015, 2016, 2017, 2018a, 2018b). Here, the essentials of limiting velocity bicubic equation elements, convenient for this discussion are from Śoln (2014, 2018a, 2018b) since these contain both positive and negative masses, $m_{+}=m \geq 0, m_{-}=-m \leq 0$, or as $m_{+,-}=m_{ \pm}= \pm m, m \geq 0$. The global adjustable values of the congruent parameter, $z(m)$,which, as such, may be even quantized, and the cubic equation discriminant, $D(m)$, affect the limiting velocity solutions with preferred energies. For instance, in Śln (2014, 2018a, 2018b), the congruent parameter, $z(m)$, and the discriminant, $D(m)$, satisfied $z^{2}(m)<1$ and $D(m)<0$, respectively, which correspond to rather high particle energies, $E \gg m v^{2}$, with $v$ denoting ordinary particle velocity. In what follows we wish to discuss lower energy regimes with light ordinary or dark matter particles, in which some energies and velocity squares may become complex. In these discussions the congruent parameter, $z(m)$, assumes specific "quantized" values of +1 ad -1 , assuring the real values of final $E$ and $v^{2}$. This is possible because, as seen in (1.2), the real and imaginary $E$ and $v^{2}$ values are respectively proportional to each other. The bicubic equation for particle limiting velocity, $c$, with global particle mass, 
$m$, and energy, $E$, is given in (1. 1),

$$
\left(\frac{c^{2}}{v^{2}}\right)^{3}-\left(\frac{E}{m v^{2}}\right)^{2}\left(\frac{c^{2}}{v^{2}}\right)+\left(\frac{E}{m v^{2}}\right)^{2}=0 .
$$

The aim of this article is to process the chosen physical parameters so as to make the particle energies, velocity squares and limiting velocities real as shown in (1.2). In the course of this process, the quantization of the particle mass, $m$, into positive mass, $m_{+}$, and negative mass, $m_{-}$, occurs which, in turn, is affecting he particle velocity square, $v^{2}$, and particle energy, $E$, respectively with making changes $\operatorname{Re} v^{2} \longmapsto v^{2}, \operatorname{Im} v^{2} \longmapsto 0$ and, $\operatorname{Re} E \longmapsto E, \operatorname{Im} E \longmapsto 0$.

$$
\begin{aligned}
m_{+,-} & =m_{ \pm}= \pm m, m \geq 0, E=\frac{3 \sqrt{3} m_{ \pm} v^{2}}{2 z\left(m_{ \pm}\right)}, z^{2}\left(m_{ \pm}\right) \geq 1, \\
D\left(m_{ \pm}\right) & =\left(\frac{27}{8}\right)^{2} \frac{1}{z^{4}\left(m_{ \pm}\right)}\left(1-\frac{1}{z^{2}\left(m_{ \pm}\right)}\right) \geq 0 .
\end{aligned}
$$

With this selection process, the congruent parameter, $z(m)$, will get quantized as $z^{2}(m)=1, z\left(m_{ \pm}\right)= \pm 1$, so that numerically in (1.2) $z^{2}(m) \geq 1$ with $D(m) \geq 0$ are satisfied, where $m_{ \pm}= \pm m, m \geq 0$. Taking these facts into account, the following are important elements of the particle limiting velocity bicubic equation as taken from Śln (2014, 2018a, 2018b) but adjusted for new parameters. Here, one assumes that the derived real energy, $E$, will not be much larger than $m v^{2}(\operatorname{Re}$ $\left.E \geq m \operatorname{Rev}^{2}\right)$, with resulting $z^{2}\left(m_{ \pm}\right) \geq 1$ and $D\left(m_{ \pm}\right) \geq 0$ as shown in detail in (1.2).

With derived real energy, $E$, (implying also real $v^{2}$ ), the negative particle mass, $m_{-}=-m<0$, is unavoidable in this presentation which will reflect explicitly on the values of the congruent parameter, $z$, satisfying $z^{2}(m)=1$, yielding of course $z(m \pm)= \pm 1$, so that $z\left(m_{+}\right)=-z\left(m_{-}\right)=1$ with $m_{ \pm}= \pm m, m \geq 0$ and with $D\left(m_{ \pm}\right)=0$.

In Section 2 the solutions for squares of primary, $c_{1}^{2}$, obscure, $c_{2}^{2}$, and normal,$c_{3}^{2}$, particle limiting velocity solutions are given. According to Burrington (1973), $c_{1}^{2}$ and $c_{2}^{2}$ are two conjugate complex roots of the bicubic equation and, as such, with their imaginary portions affecting the particle energy and velocity-square to be complex. Adjusting the particle parameters so as to make imaginary portions of the energy and velocity squared simultaneously equal to zero, $\operatorname{ImE}=0$, $\operatorname{Im} v^{2}=0$, one obtains that $c_{1}^{2}$ and $c_{2}^{2}$ become numerically the same limiting velocity squares of $m_{+}=m$ and $m=-m$, $m \geq 0$ massive particles. Although the square of normal,limiting velocity satisfies $c_{3}^{2} \prec 0$, and implying the $c_{3}$ to be imaginary, the corresponding particle, never the less, has real ordinary velocity and the same real energy as particle with either $c_{1}^{2}$ or $c_{2}^{2}$ limiting velocity squares. A particle with a real ordinary velocity, $v$, and real energy, $E$, but imaginary limiting velocity $c_{3}$, making it difficult to identify, might be a potential example of a dark matter particle. In fact, the interest today is rather strong to explore large ranges of dark matter particles from ultraviolet to sub-MeV in energies (see, for instance Alonso, Blas, \& Wolf, 2018). Hence, here in this work, we wish to address the behaviors of light ordinary or dark matter particles and see how these can be used when applied to the light sterile neutrino dark matter particles $\mathrm{Ng}$ et al. (2019).

Section 3 is devoted to summary with discussion and the treatment of specific examples of sterile neutrino dark matter particles.

\section{Limiting velocity solutions with positive and negative particle masses}

The mathematical solutions (Burrington, 1973) for the squares of primary, obscure and normal limiting velocities, $c_{1}^{2}, c_{2}^{2}$ and $c_{3}^{2}$, are derived from (1.1) as follows,

$$
\begin{aligned}
\frac{c_{1,2}^{2}(m)}{v^{2}}= & \frac{3}{2 z(m)} \csc 2 \tan ^{-1}\left(\tan \left(\frac{1}{2} \sin ^{-1}\left(\frac{1}{z(m)}\right)\right)\right)^{\frac{1}{3}} \\
& \pm i \frac{3 \sqrt{3}}{2 z(m)} \operatorname{ctn} 2 \tan ^{-1}\left(\tan \left(\frac{1}{2} \sin ^{-1}\left(\frac{1}{z(m)}\right)\right)\right)^{\frac{1}{3}} \\
c_{1,2}= & c_{1}, c_{2}: c_{1} \# c_{2} \\
\frac{c_{3}^{2}(m)}{v^{2}}= & -\frac{3}{z(m)} \csc 2 \tan ^{-1}\left(\tan \left(\frac{1}{2} \sin ^{-1}\left(\frac{1}{z(m)}\right)\right)\right)^{\frac{1}{3}},
\end{aligned}
$$

where in $(2.1,2)$ for $c_{1,2}$, at this stage the inequality still holds between limiting velocities $c_{1}$ and $c_{2}$. Hence, it is easily seen that $c_{1}^{2}+c_{2}^{2}+c_{3}^{2}=0$. While mathematically, these solutions are satisfactory, physically, as yet, they are not. From 
(1.2) we have for the particle energy expression $E=3 \sqrt{3} m v^{2} / 2 z(m)$, so that with $v^{2}$ from $(2,1,2)$ and $(2.3)$ each energy can be expressed directly in terms of $c_{1}^{2}, c_{2}^{2}$ and $c_{3}^{2}$ yielding two complex energy expressions and one real. For further easier discussions of energy relations it is worthwhile introducing the following notation,

$$
\alpha(m)=2 \tan ^{-1}\left(\tan \left(\frac{1}{2} \sin ^{-1}\left(\frac{1}{z(m)}\right)\right)\right)^{\frac{1}{3}} .
$$

From relations $(2,12)$ and (2. 3) one can express the energy with each particle limiting velocity as,

$$
E\left(c_{i}\right)=\frac{3 \sqrt{3} m v^{2}\left(c_{i}\right)}{2 z(m)}, i=1,2,3,
$$

and, after expressing $v^{2}\left(c_{i}\right), i=1,2,3$ in terms of $c_{1,2}^{2}(m)$ and $c_{3}^{2}(m)$ yielding from $(2.1,2)$ and $(2.3)$ the multiple energy expressions, labeled by limiting velocities,

$$
\begin{gathered}
E\left(c_{1,2}\right)=\sqrt{3} m c_{1,2}^{2}(m)[\csc \alpha(m) \pm i \sqrt{3} \operatorname{ctn} \alpha(m)]^{-1} \\
E\left(c_{3}\right)=-\frac{\sqrt{3}}{2} m c_{3}^{2}(m)[\csc \alpha(m)]^{-1}
\end{gathered}
$$

The simple question now is, what values of physical parameters will make the energies $E\left(c_{1}\right)$ and $E\left(c_{2}\right)$ real without imaginary portions ? To begin with, the imaginary portions in either $(2.1,2)$ or $(5.1,2)$ disappear when $\alpha(m)= \pm \pi / 2$. These values of $\alpha(m)$ quantize the congruent parameter, $z(m)$, into $z(m \pm)= \pm 1$ and the mass, $m$, into the positive mass, $m_{+}=m$, and the negative mass, $m_{-}=-m, m \geq 0$, through the following chain of deductions,

$$
\begin{aligned}
& \left(\alpha(m)=2 \tan ^{-1}\left(\tan \left(\frac{1}{2} \sin ^{-1}\left(\frac{1}{z(m)}\right)\right)\right)^{\frac{1}{3}}= \pm \frac{\pi}{2}\right. \\
& \operatorname{ctn} \alpha(m)=\operatorname{ctn}\left( \pm \frac{\pi}{2}\right)=0, \csc \alpha(m)= \pm 1 \longrightarrow \\
& \csc 2 \tan ^{-1}\left(\tan \left(\frac{1}{2} \sin ^{-1}\left(\frac{1}{z(m)}\right)\right)^{\frac{1}{3}}= \pm 1\right. \\
& \tan ^{-1}\left(\tan \left(\frac{1}{2} \sin ^{-1}\left(\frac{1}{z(m)}\right)\right)^{\frac{1}{3}}= \pm \frac{\pi}{4}\right. \\
& \tan \left(\frac{1}{2} \sin ^{-1}\left(\frac{1}{z(m)}\right)\right)=( \pm 1)^{3}= \pm 1 \\
& \frac{1}{2} \sin ^{-1}\left(\frac{1}{z(m)}\right)= \pm \frac{\pi}{4}, \frac{1}{z\left(m_{ \pm}\right)}= \pm 1 \\
& \csc \alpha\left(m_{ \pm}\right)= \pm 1=z\left(m_{ \pm}\right), m_{ \pm}= \pm m, m \geq 0 \\
& z\left(m_{ \pm}\right)=\frac{3 \sqrt{3} m_{ \pm} v^{2}}{2 E\left(m_{ \pm}\right)}= \pm 1: E\left(m_{ \pm}\right)=\frac{3 \sqrt{3} m v^{2}}{2} \text {. }
\end{aligned}
$$

As one sees, these deductions not only eliminated the imaginary parts from energies $E\left(c_{1}\right)$ and $E\left(c_{2}\right)$ but also from limiting velocity squares, $c_{1}^{2}\left(m_{ \pm}\right)$and $c_{2}^{2}\left(m_{ \pm}\right)$. One should notice the appearance of the energy, $E\left(m_{ \pm}\right)=3 \sqrt{3} m v^{2} / 2$, in (6.3) which, numerically, it will turn out not only to be $E\left(c_{1}\right)$ and $E\left(c_{2}\right)$ but also $E\left(c_{3}\right)$. In other words, the same energy for all three particle with limiting velocities, $c_{i}, i=1,23$. Also,important thing to notice is the fact that these deductions in a simple way quantized the congruent parameter, $z\left(m_{ \pm}\right)$, into \pm 1 values which, in turn quantizes he mass into $m_{ \pm}= \pm m$ with $m \geq 0$. . Furthermore, with parameter values from relations $(6.1,2,3)$, the primary, obscure and normal limiting velocity squares follow from $(2.1,2)$ and $(2.3)$ to be 


$$
\begin{aligned}
c_{1,2}^{2}\left(m_{ \pm}\right) & =c_{1}^{2}\left(m_{ \pm}\right)=c_{2}^{2}\left(m_{ \pm}\right)=\frac{3 v^{2}}{2 z\left(m_{ \pm}\right)} \csc \alpha\left(m_{ \pm}\right)=\frac{3}{2} v^{2}, \\
c_{3}^{2}\left(m_{ \pm}\right) & =-\frac{3 v^{2}}{z\left(m_{ \pm}\right)} \csc \alpha\left(m_{ \pm}\right)=-3 v^{2} .
\end{aligned}
$$

At this point one notices that numerically $c_{1}^{2}\left(m_{ \pm}\right)$and $c_{, 2}^{2}\left(m_{ \pm}\right)$are equal to each other for either $m_{+}=m$ or $m_{-}=-m$ with $m \geq 0$ simply because the imaginary portions of energies are eliminated by choices of parameters in (6.1), (6.2) and (6.3). One retains notation $c_{1,2}^{2}\left(m_{ \pm}\right)$which may allow to differentiate between $c_{1}$ and $c_{2}$ with some other possible attributes, if and when necessary.

Next, we demonstrate that $E\left(m_{ \pm}\right)=3 \sqrt{3} m v^{2} / 2$ from (6.3) is universal. Namely, as suggested by (6.1) and (5.2), one starts with real portions of $(5.1,2)$ and $(5.3)$, and then with the same choice of parameters from $(6.1,2 ; 3)$ obtains for particle energies with primary, obscure and normal limiting velocities for either $m_{+}=m$ or $m_{-}=-m$ with $m \geq 0$, the following expressions (with $\csc \alpha\left(m_{ \pm}\right)=z\left(m_{ \pm}\right)= \pm 1$ ):

$$
\begin{aligned}
E\left(c_{1,2}\left(m_{ \pm}\right)\right) & =\sqrt{3} m_{ \pm}\left(\csc \alpha\left(m_{ \pm}\right)\right)^{-1} c_{1,2}^{2}\left(m_{ \pm}\right) \\
& =m_{ \pm}( \pm 1) \frac{3 \sqrt{3}}{2} v^{2}=m \frac{3 \sqrt{3}}{2} v^{2} . \\
E\left(c_{3}\left(m_{ \pm}\right)\right) & =-\frac{\sqrt{3}}{2} m_{ \pm}\left(\csc \alpha\left(m_{ \pm}\right)\right)^{-1} c_{3}^{2}\left(m_{ \pm}\right) \\
& =m_{ \pm}( \pm 1) \frac{3 \sqrt{3}}{2} v^{2}=m \frac{3 \sqrt{3}}{2} v^{2} .
\end{aligned}
$$

Indeed, this same numerical energy is associated with three particles, having the same $m_{+}$or $m_{-}$masses and the same ordinary velocity, $v$, but two different limiting velocities, one real $c_{1,2}$ and one imaginary $c_{3}$. This last fact, makes these particles rather difficult to associate with each other. Are the so called dark matter particles from this class of particles?

Utilizing the connections between particle ordinary velocity, $v$, and the single valued doublet, $c_{1,2}$, limiting velocity or, $c_{3}$, limiting velocity in $(7.1,2 ; 3)$ one re-expresses particle energies from $(8.1,2 ; 3)$ in the bicubic equation limiting particle velocity formalism with multiple limiting velocities in fixed Lorentzian like forms from reference (Śoln, 2017) as follows,

$$
\begin{aligned}
E\left(c_{1,2}\left(m_{ \pm}\right)\right) & =\frac{3}{2} m_{ \pm}( \pm 1) v^{2}\left(1-\frac{v^{2}}{c_{1,2}^{2}\left(m_{ \pm}\right)}\right)^{-\frac{1}{2}}=\frac{3}{2} m v^{2}\left(1-\frac{v^{2}}{c_{1,2}^{2}\left(m_{ \pm}\right)}\right)^{-\frac{1}{2}}, \\
v^{2} & =\frac{2}{3} c_{1,2}^{2}\left(m_{ \pm}\right)>0 . \\
E\left(c_{3}\left(m_{ \pm}\right)\right) & =3 m_{ \pm}( \pm 1) v^{2}\left(1-\frac{v^{2}}{c_{3}^{2}\left(m_{ \pm}\right)}\right)^{-\frac{1}{2}}=3 m v^{2}\left(1-\frac{v^{2}}{c_{3}^{2}\left(m_{ \pm}\right)}\right)^{-\frac{1}{2}}, \\
v^{2} & =-\frac{1}{3} c_{3}^{2}\left(m_{ \pm}\right)>0 .
\end{aligned}
$$

One thing that one sees immediately from $(9.1,2 ; 3)$ is that these forms for the energy expressions are very accommodating. Here, consistent with (6.3), we have particles with the same energy $E$, the same mass $m_{+}$or $m_{-}$with the same velocity $v$; along with a single valued doublet limiting velocity, $c_{1,2}$, and the other with imaginary value, $c_{3}$, limiting velocity. In other words, while the particle velocity, $v$, is fixed, the limiting velocities are not.The limiting velocities although being so different, nevertheless steer the particle in question to the same energy and with the same particle velocity, $v$. One also should notice that all these energies coming out with the same value are consistent with same congruent parameter values of, $z\left(m_{ \pm}\right)= \pm 1$,in relations $(5.1,2 ; 3)$ and $(8.1,2 ; 3)$ even before going into details of determinations of energies with limiting velocities.

\section{Discussion, one example and conclusion}

Although, the energy expressions in $(9.1,2 ; 3)$ have form similarities, on one hand, with the Lorentzian particle energy expression in the theory of relativity (Alonso, Blas, \& Wolf, 2018) with the velocity of light, $c$, as the single limiting velocity, it is, on the other hand, the bicubic equation limiting particle velocity formalism (Śoln, 2017) with multiple limiting velocities, $c_{1}, c_{2}$ and $c_{3}$, that yield the Lorentzian like particle energy expressions with fixed particle velocity, $v$, but with interchangeable limiting velocities, $c_{1,2}$ and $c_{3}$. A rather interesting and intriguing fact is that a particle with fixed 
ordinary velocity can change the limiting single valued doublet real velocity, $c_{1,2}$, into the imaginary velocity, $c_{3}$, and still retain the same energy, $E$.

We can actually, give an outline of the real and imaginary limiting velocities occurrences on an example from the literature. Namely,recently Kenny C. Y. Ng et al. (2019) in studying the constraints on sterile neutrino dark matter from NuSTAR M31 observations, have put forward a well-motivated sterile neutrino dark matter candidate denoted as $\chi$. This well studied sterile neutrino candidate can radioactively decay into mono-energetic photon, $\gamma$, and into an active neutrino, $\nu$. It was concluded that the mass of the sterile neutrino satisfy $m_{\chi} c^{2} \geq 12 \mathrm{keV}$, with $c$ the velocity of light. Here, it may be assumed that the sterile neutrino dark matter particle physical parameters nature chose so as to only real portion of the energy remains. If so, this sterile neutrino dark matter particle should through limiting velocities, the real one, $c_{1,2}$, and the imaginary,$c_{3}$, obey the same value energy expressions $(8.1,2 ; 3)$, allowing the masses to be positive and negative. For simplicity we choose that $c_{1,2}=c$, the velocity of light, and that $m_{\chi} c^{2}=12 \mathrm{keV}$. Then, consistent with $(7.1,2 ; 3)$ we can immediately write, with the help from $(8.1,2 ; 3)$ :

$$
\begin{aligned}
( \pm 1) m_{\chi}^{ \pm} c^{2} & =m_{\chi} c^{2}=12 \mathrm{keV}: \\
\text { Def. } & : \quad c_{1,2}=c ; v^{2}=\frac{2}{3} c_{1,2}^{2}\left(m_{\chi}^{ \pm}\right)=\frac{2}{3} c^{2} \hookleftarrow c_{3}^{2}\left(m_{\chi}^{ \pm}\right)=-2 c^{2}, \\
E\left(c_{1,2}\left(m_{\chi}^{ \pm}\right)\right) & =( \pm 1) m_{\chi}^{ \pm} \frac{3 \sqrt{3}}{2} v^{2}=\sqrt{3} m_{\chi} c^{2}=\sqrt{3} 12 \mathrm{keV}=20.8 \mathrm{keV}, \\
E\left(c_{3}\left(m_{\chi}^{ \pm}\right)\right) & =-\frac{\sqrt{3}}{2}( \pm 1) m_{\chi}^{ \pm} c_{3}^{2}\left(m_{ \pm}\right)=\sqrt{3} m_{\chi} c^{2}=\sqrt{3} 12 \mathrm{keV}=20.8 \mathrm{keV},
\end{aligned}
$$

Both particles, one with real limiting velocity, $c_{1,2}$, and the other with imaginary limiting velocity, $c_{3}$, have the same energy, something that it should not be too surprising for particles belonging to the dark matter species.

In conclusion one may say that,on one hand, consistent with our present understanding,some portions of the nature appear to be rather simple and predictable, but on the other hand, some portions of the nature can also be rather complex, indicating that some answers are still at a distance and eventually to be uncovered,leading to better understanding.

\section{References}

Alonso, R., Blas, D., \& Wolf, P. (2018). Exploring the ultra-light to sub-MeV dark matter window with atomic clocks and co-magnetometers. arXiv preprint arXiv:1810.00889.

Burrington, R. S. (1973). Handbook of Mathematical Tables and Formulas. New York: IMcGraw-Hill.

Śoln, J. (2014). Theoretical particle limiting velocity from the bicubic equation: Neutrino example. Physics Essays, 27(3), 448-453.

Śoln, J. (2015). Particle limiting velocities from the bicubic equation derived from Einstein's kinematics: PeV electron case. Applied

Physics Research, 7(4), 37.

Śoln, J. (2016). Limiting velocities of primary, obscure and normal particles: Self-annihilating obscure particle as an example of dark matter particle. Applied Physics Research, 8(5), 1.

Śln, J. (2017). Connecting dark matter particles with the primary, obscure and normal particles through implicit causality. Applied Physics Research, 9(3), 1.

Śln, J. (2018a). Positive and negative particle masses in the bicubic equation limiting particle velocity formalism. Applied Physics Research, 10(1), 14.

Śoln, J. (2018b). Similarities and differences between positive and negative particle masses in the bicubic equation limiting particle velocity formalism: positive or negative muon neutrino mass? Applied Physics Research, $10(5), 40$.

Ng, K. C., Roach, B. M., Perez, K., Beacom, J. F., Horiuchi, S., Krivonos, R., \& Wik, D. R. (2019). New Constraints on Sterile Neutrino Dark Matter from NuSTARM31 Observations. arXiv preprint arXiv:1901.01262.

\section{Copyrights}

Copyright for this article is retained by the author(s), with first publication rights granted to the journal.

This is an open-access article distributed under the terms and conditions of the Creative Commons Attribution license (http://creativecommons.org/licenses/by/4.0/). 